\title{
La enfermedad neurológica de Leonardo da Vinci
}

\author{
The neurological disease of Leonardo da Vinci
}

Patricio Sandoval Rubio ${ }^{1}$

\begin{abstract}
Resumen
Introducción: el año 2019 se conmemoraron los 500 años de la muerte de Leonardo da Vinci en el Chateux du Cloux, Francia. Según registros históricos, Leonardo vivió los últimos años de su vida con una parálisis en su mano derecha, atribuida a un probable accidente cerebrovascular (ACV), lo cual afectó a su capacidad para emprender nuevos proyectos pictóricos. Este trabajo describe como fueron los últimos años de vida de Leonardo, revisa los antecedentes sobre salud y su probable enfermedad neurológica, sus eventuales etiologías y sus consecuencias. Desarrollo: Leonardo llegó a Francia en 1516 por invitación del rey Francisco I para llevar el Renacimiento y completar su formación personal. En 1517 es visitado por el cardenal Luis de Aragón, y su secretario registra en su diario que Leonardo se encuentra afectado de una parálisis de la mano derecha. Algunos autores sugieren un ACV como causa probable, sobretodo porque según el historiador Vasari, Leonardo vivió sus últimos años en condición de discapacidad. Se llega a plantear que su condición de vegetariano habría podido influir como factor de riesgo de ACV. Sin embargo, un dibujo no fechado de Figino muestra a Leonardo con una parálisis de tipo periférica de la mano derecha, abriendo el diagnóstico diferencial. Conclusiones: no hay información suficiente para valorar el tipo y la causa de enfermedad neurológica de Leonardo da Vinci, no obstante, está influyó significativamente en su interacción con su discípulo Francisco Melzi y su mecenas Francisco I, hecho que contribuyó a que su legado perdure hasta hoy.
\end{abstract}

Palabras clave: Leonardo da Vinci; parálisis, accidente cerebrovascular; veganismo; enfermedad de neurona motora; metales pesados.

\begin{abstract}
Introduction: the year 2019 marked the 500th anniversary of the death of Leonardo da Vinci at the Chateau du Cloux, France. According to historical records, Leonardo lived the last years of his life with palsy on his right hand, attributed to a probable stroke, which affected his ability to undertake new pictorial projects. This study describes how were the last years of Leonardo's life, reviews his medical history and his probable neurological disease, with its possible etiologies and its consequences. Discussion: Leonardo arrived in France in 1516 invited by King Francis I to lead the Renaissance and complete his education. In 1517, cardinal Luis de Aragón visited Leonardo, and his secretary recorded in his diary that he was affected by a palsy on his right hand. Some authors suggest that stroke could have been a probable cause, especially because according to the historian Vasari, Leonardo lived his last years in a condition of disability. His vegetarian food habit could have been a risk factor for his suspected stroke. However, an undated drawing by Figino shows Leonardo with a peripheral nerve palsy on his right hand, opening up the differential diagnosis. Conclusions: there is not enough information to assess the type and cause of Leonardo da Vinci's neurological disease. However, this condition significantly affected his interaction with his disciple Francisco Melzi and his patron Francisco I, a fact that contributed to his legacy enduring until today.
\end{abstract}

Keywords: Leonardo da Vinci; palsy, stroke; veganism; motor neuron disease; heavy metals.

Fecha de envío: 2020-09-09 - Fecha de aceptación: 2021-03-16

\section{Introducción}

El 2 de mayo del año 2019 se celebraron los 500 años de la muerte de Leonardo da Vinci (1452 - 1519). El mundo de la ciencia, la cultura y las artes, le conmemoró con múltiples exposiciones, actos y publicaciones, reivindicando su legado y trascendencia (Bambach, 2019; Remuzzi \& Ricciardi, 2019; Tambone, 2019).

(1) Departamento de Neurología. Escuela de Medicina, Facultad de Medicina, Pontificia Universidad Católica de Chile Autor de correspondencia: psandova@med.puc.cl 
Leonardo, prototipo del homo univerisalis, terminó sus días en el Chateau du Clos - Lucé en el valle de Loira, invitado por el rey de Francia (Pedretti, 2011), en cuyos brazos según la tradición, falleció (Nicholl, 2005; Vasari, 2012; Isaacson, 2017). Lo que es menos conocido es que en sus últimos años estuvo afectado de lo que parece haber sido una enfermedad neurológica progresiva la cual ha sido atribuida a accidentes cerebrovasculares (ACV) recurrentes (Coralli \& Perciaccante, 2016).

El presente trabajo tiene por objetivo relatar como fueron los últimos días de Leonardo en Francia, como era su condición de salud, analizar que antecedentes hay que sustenten la hipótesis del ACV, sus eventuales causas, diagnósticos diferenciales y el impacto que tuvo su patología neurológica en su obra y en su legado.

\section{Contexto histórico}

En el mes de diciembre de 1515, Leonardo forma parte de la comitiva papal que se reunió en Bolonia con el nuevo rey de Francia, Francisco I (1494 - 1547). Leonardo había llegado a Roma dos años antes, a petición de Juliano Il de Medicis, quien asumió con confaloniero de su hermano Giovani, recién nombrado Papa con el nombre de León X (Nicholl, 2005; Isaacson, 2017). Así, los hijos de Lorenzo el Magnífico saldaban la deuda con Leonardo por la indiferencia con la que su padre le trató (Isaacson, 2017). No obstante, en Roma dominaban Bramante, Rafael, y su némesis, Miguel Ángel Buonarroti, por lo que Leonardo, con más de 60 años, se limitó a realizar estudios científicos más que proyectos artísticos (Nicholl, 2005; Isaacson, 2017). En ese contexto, el encuentro con el nuevo rey de Francia, un joven de 21 años muy educado y ávido de conocimiento, debió haber sido visto por Leonardo como una oportunidad para encontrar un nuevo mecenas que estuviese a la altura de sus talentos y de su momento biográfico. Francisco I, admirador de Leonardo y futuro coleccionista de sus obras, acababa de conquistar Milán y se reunió con el Papa para negociar una poco duradera paz que quedó sellada en el Concordato de Bolonia (1516). Estimulado por su madre Luisa de Saboya, Francisco I deseaba desarrollar el Renacimiento en Francia, por lo que invita a Leonardo a ser parte de su corte (Nicholl, 2005; Pedretti, 2011; Isaacson, 2017).

No sabemos en qué términos y plazos fueron esas negociaciones, pero la muerte Juliano II de Medicis de tuberculosis el 17 de marzo de 1516, aceleró la decisión (Nicholl, 2005; Isaacson, 2017). Una carta fechada cuatro días antes, enviada por el confidente de Francisco I, Guillaume Gouffier, al embajador de Francia en Roma, Antonio María Pallavicini, reafirma la invitación previamente extendida (Pedretti, 2011).

Lo siguiente que sabemos es que, en el verano de 1516, antes de que la nieve invernal impidiera el cruce de Los Alpes, Leonardo emprende su último viaje, esta vez a Francia, en compañía de su discípulo Francesco Melzi, su sirviente Battista de Vilanis y sus inseparables cuadros "Santa Ana, la Virgen y el Niño", "San Juan Bautista" y la "Mona Lisa" (Nicholl, 2005; Pedretti, 2011; Isaacson, 2017).

En Francia, Leonardo recibe un generoso estipendio y la libertad de crear e investigar a su propia voluntad. Adicionalmente, se le facilita el Chateau du Cloux (hoy Clos - Lucé), a menos de un kilómetro del palacio real de Amboise en el valle del rio Loira (Nicholl, 2005; Pedretti, 2011; Isaacson, 2017). Sus únicas labores formales fueron rediseñar la ciudad de Romorantín, un ambicioso proyecto que consideraba un complejo arquitectónico de edificios y canales, y organizar eventos para el rey en las celebraciones oficiales (Nicholl, 2005; Pedretti, 2011; Isaacson, 2017). Sin embargo, la mayor parte del tiempo le fue demandado en completar la formación de Francisco, con quien tenía largas tertulias de los más variados temas. Francisco I llegó a expresar a Benvenuto Cellini que, "no creía que hubiera nacido nunca nadie que supiera tanto como Leonardo, y no solo en los campos de la pintura, la escultura y la arquitectura, sino que también pensaba que era un gran filósofo" (Isaacson, 2017).

\section{La visita del cardenal Luis de Aragón}

En octubre de 1517, Leonardo es visitado en su taller por el cardenal Luis de Aragón. Su secretario, Antonio de Beatis, registra en su diario de viaje: "un tipo de parálisis le ha invalidado su mano derecha...y aunque ya no puede pintar con el talento que le era característico, aún puede diseñar y enseñar a otros" (Nicholl, 2005; Isaacson, 2017). La afirmación sorprende ya que es bien conocido no solo que Leonardo escribía de manera especular con la mano izquierda, sino que por la dirección del achurado de las sombras en sus dibujos, se ha determinado que también pintaba con la mano izquierda (Scott, 1979; Reinke, 1993; Bambach, 2003; McMannus \& Drury, 2004; King, 2016). Existen además antecedentes de que hizo retoques a los tres cuadros que conservaba posterior a la visita del cardenal de Aragón (Bambach, 2019). Esto hace sospechar que, si bien la descripción de Antonio de Beatis hace referencia a una monoparesia braquial, una posibilidad es que debió haber estado afectado de una hemiparesia derecha o de una condición que le dificultaba globalmente su movilidad, impidiéndole sostener una paleta de pinturas e imposibilitándole acometer nuevos y grandes proyectos pictóricos, pero no hacer retoques sentado con su mano izquierda a los cuadros que conservaba y que estimaba inconclusos.

\section{El dibujo de Figino}

Un dibujo atribuido a Giovanni Ambrogio Figino de fecha no determinada, muestra a un Leonardo con la mano derecha contenida por sus ropas a la manera de un cabestrillo, en una posición distinta a la esperable para una secuela de un ACV (figura 1)(Lazzeri \& Rossi, 
2019). Esto es, en vez de una hipertonía de los músculos flexores con inclusión del pulgar, se observa la mano abierta, con un aspecto hipotónico, donde los dedos cuarto y quinto se encuentran en semiflexión, lo que ha hecho plantear como diagnósticos alternativos una lesión de nervio ulnar, una distonía o incluso una contractura de Dupuytren (De Campos et al., 2019). Destacablemente, en el dibujo de Figino, Leonardo no muestra signos de una parálisis facial central derecha, lo que habría apoyado la hipótesis de la hemiparesia y a su vez del ACV. No obstante, esto bien podría ser por que la paresia facial fuese sutil, por una lesión cortical que hubiese afectado solo el área de representación de la mano, o por un mal registro del artista.

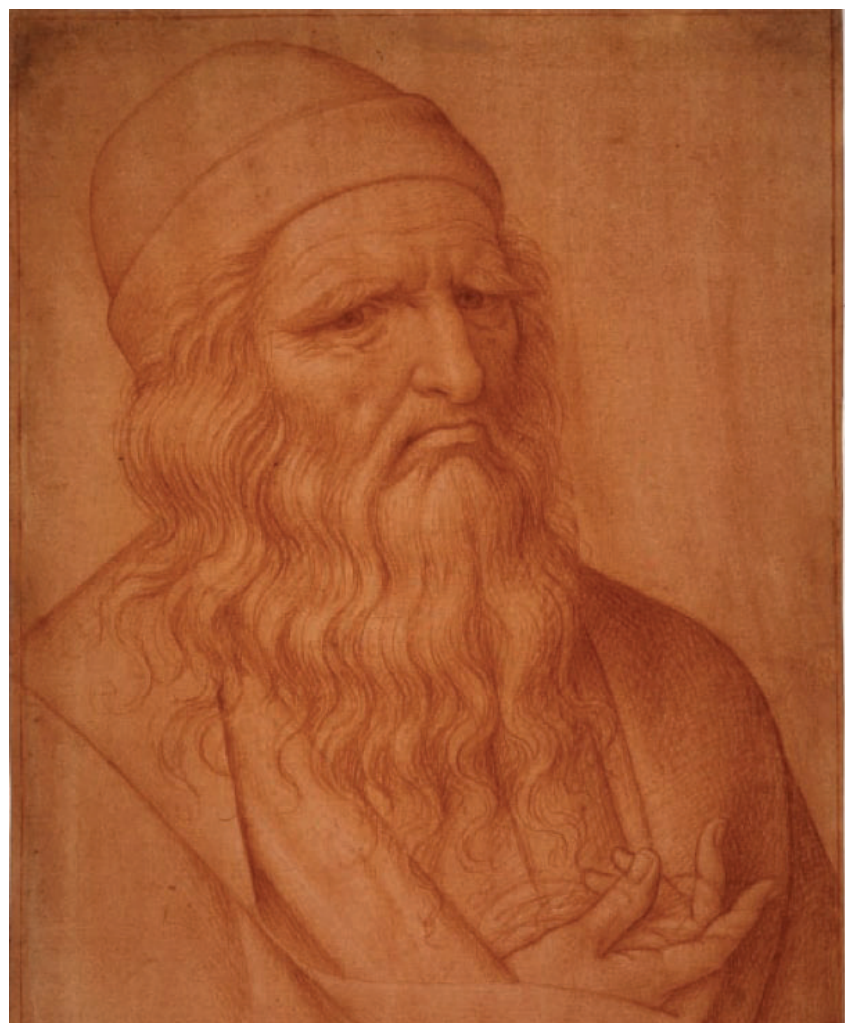

Figura 1: Retrato de Leonardo da Vinci, Giovanni Ambrogio Figino (fecha no precisada, Siglo XVI): Este retrato muestra a un Leonardo envejecido, poniendo en evidencia la parálisis de su mano derecha. La mano adopta una postura más sugerente de una lesión de nervio ulnar ("mano del predicador") que de una secuela espástica propia de una lesión piramidal por un ACV. [Galería de la Academia de Venecia. Italia. Archivo fotográfico, Ministerio de bienes y actividades culturales y turísticas].

\section{El relato de Giorgio Vasari}

Según Giorgio Vasari, el artista e historiador del Renacimiento, en su célebre obra "Las vidas de los más excelentes pintores, escultores y arquitectos" (1550), Leonardo estuvo enfermo "por muchos meses", "no podía pararse" y requería "ser sostenido por los brazos de sus sirvientes y sus amigos" (Vasari, 2012). Esta imagen nos revela a un
Leonardo bastante más postrado que lo relatado por Antonio de Beatis en 1517, sugiriendo que pudo haber sufrido ACVs recurrentes (Coralli, 2016). Incluso, Vasari nos dice que Leonardo murió de un "paroxismo"(Vasari, 2012), lo que hoy puede ser entendido como un ACV, una convulsión o una muerte súbita de causa cardiovascular. Tanto Vasari como de Beatis no describen deterioro cognitivo ni del lenguaje, lo que también habría apoyado una localización en el sistema nervioso central de su déficit neurológico.

Otra posibilidad, que debe considerarse en una enfermedad progresiva que provoca una parálisis de una mano sin alteración cognitiva ni de lenguaje (al menos inicialmente), es una enfermedad de la neurona motora.

\section{La dieta vegetariana y el riesgo de ACV}

Jean Paul Richter, quien fuese el primero en descifrar y traducir los manuscritos de Leonardo a fines del siglo XIX, hizo explícito que Leonardo fue lo que hoy llamaríamos animalista y vegetariano. Richter manifiesta "Nos inclinamos a pensar que el propio Leonardo era vegetariano", esto basado en una carta enviada desde la India por el explorador florentino Andrea Corsali a Juliano II de Medicis, en la que describe que"ciertos infieles llamados guzzarati [guyaratis] no se alimentan de nada que contenga sangre, ni permiten entre ellos infligir daño a ninguna criatura viviente, como nuestro Leonardo da Vinci" (Richter, 1977). Así, una vez más, Leonardo se anticipó en siglos a su época (Muntz, 1898).

Algunos autores han atribuido a su condición de vegetariano ser un factor de riesgo para el presunto ACV de Leonardo (Özturk, 2009; Özturk et al., 2010).

La relación entre dieta basada en vegetales y el riesgo de ACV es controvertida, ya que si bien esta favorece significativamente el control de múltiples factores de riesgo vascular (Campbell, 2017), algunos estudios han sugerido que un aumento en la homocisteína y una disminución de los niveles IGF-1 podrían dañar el endotelio vascular y aumentar el riesgo (McCarty, 2003). Las dietas vegetarianas provocan una deficiencia de vitamina B12 con la consecuente acumulación de homocisteína, cuyos niveles elevados se han asociado a una mayor recurrencia de infarto cerebral (Shi et al., 2018).

Recientemente, el estudio de cohorte prospectivo EPIC - Oxford, con más de cuarenta y ocho mil pacientes y 18 años de seguimiento, encontró que si bien el riesgo de infarto al miocardio es menor en pacientes veganos, el de ACV global (isquémico y hemorrágico) y el de ACV hemorrágico, son mayores (Tong et al., 2019). Esto último, como hipótesis, por la relación entre niveles bajos de colesterol plasmático con un mayor riesgo de hemorragia intracerebral (Ma et al., 2019). 
Hasta la fecha no existen meta-análisis ni estudios randomizados que respondan esta controversia, por lo que hasta ahora, se sugiere seguir las recomendaciones nutricionales de la American Heart Association (AHA/ASA) para prevención primaria y secundaria (Kernan et al., 2014; Meschia et al., 2014).

\section{La salud de Leonardo}

Leonardo siempre se preocupó de su salud. Tenía libros sobre cómo mantenerse saludable y en buena forma física (King, 2016). Gustaba de practicar el montañismo y era famoso por su prestancia (Vasari, 2012; Bambach, 2019). Como vimos en el párrafo anterior, era vegetariano (Richter, 1977), lo que recientemente se ha vinculado a mayor longevidad (Nagashi et al., 2020).

No hay antecedentes de enfermedades en sus notas ni cartas hasta el año 1515, en que en una carta a Juliano II de Medicis le reporta que se encuentra enfermo (King, 2016). No hace mención a los síntomas ni a su gravedad, no obstante, a pesar de desconfiar de los médicos y medicinas de la época (probablemente por lo poco científico que le parecían sus conocimientos y procedimientos), ese mismo año aparece vinculado a un médico de nombre Gaiacqo, quien oficia como su patrocinador para ingresar a la cofradía San Juan de los Florentinos en Roma (Nicholl, 2005; King, 2016). Una de las razones para ingresar a estas cofradías era asegurarse un digno funeral (Nicholl, 2005). También en 1515, Leonardo registra en sus notas una serie de sugerencias para mantenerse en buena salud: "no coma cuando no tenga apetito, mastique bien los alimentos, use ingredientes simples, no tome medicamentos, no duerma a la mitad del día, mezcle el vino con agua, no haga ejercicio extenuante, y evite el desenfreno" (King, 2016, Campanozzi et al., 2020).

Todo lo anterior indica que efectivamente se sentía enfermo e intuía que se encontraba en el inicio de su fin. En sus cuadernos de notas, escribió: ¡Oh tiempo! consumidor de todas las cosas; ¡Oh edad envidiosa! destruyes todas las cosas y las devoras con los implacables dientes de los años, poco a poco en una muerte lenta (Richter, 1977).

A pesar del uso de productos tóxicos en la pintura como el "blanco de plomo", o arsénico y mercurio en sus experimentos en busca de nuevos pigmentos, no hay registro de que haya tenido síntomas evidentes que sugirieran una intoxicación crónica por metales pesados (King, 2016). Sin embargo, la exposición a metales pesados se ha relacionado, de manera no categórica, a una mayor incidencia de enfermedad de la neurona motora (Riancho et al., 2018). Tampoco hay reportes de síntomas neurológicos ni sistémicos atribuibles a carencia de vitamina B12, considerando su condición de vegetariano.
Leonardo vivió 67 años, en una época en que la esperanza de vida era de aproximadamente 40 años para la población general (Peller, 1947) y 54 años para los hombres de familias nobles europeas (Cummins et al., 2017). No obstante, su sobrevida fue cercana a otros artistas de la época (McManus, 1975; Greenspan et al., 2008), aunque mucho más que la de Rafael Sanzio quien falleció a los 37 años (Forcellino, 2012) y bastante menos que la de Miguel Ángel Buonarroti, quien vivió hasta los 88 años (Wallace, 2011). El padre de Leonardo, Piero da Vinci y su presunta madre, Caterina di Meo Lippi, fallecieron a los 78 y 57 años, respectivamente (Isaacson, 2017; Kemp \& Pallanti, 2017).

Así, Leonardo llevó una vida bastante saludable, con hábitos que contribuyeron a reducir su riesgo cardio y cerebrovascular según nuestros parámetros y recomendaciones actuales. No obstante, aun así, es imposible valorar la plausibilidad de la hipótesis del ACV al desconocer la presencia o no de factores de riesgo vascular como hipertensión arterial, diabetes mellitus o dislipidemia.

\section{La muerte y la eternidad}

El 2 de mayo del año 1519, fallece Leonardo de Vinci en su habitación en el Chateau du Cloux (Nicholl, 2005; Isaacson, 2017). Pocos días antes, el 23 de abril, Leonardo, presintiendo su muerte, había hecho su testamento en el que legaba a su discípulo Francesco Melzi los cuadros que conservaba y todos sus dibujos y cuadernos de notas. A sus sirvientes Battista de Vilanis y Gian Giacomo Caprotti (conocido como Salai), les heredó a cada uno la mitad de una viña que Ludovico Sforza le había obsequiado en Milán, mientras que a su cocinera, Maturina, un "vestido de paño negro forrado en piel" $y$ dos ducados (Nicholl, 2005; Isaacson, 2017). Luego de la muerte de Leonardo, Melzi estuvo más de 50 años ordenando sus cuadernos de notas y dibujos en la villa familiar de Vaprio d'Adda en las cercanías de Milán, llegando a publicar de manera póstuma un "Tratado de la pintura" (Bambach, 2019), aunque su propia muerte en 1570, le impediría concretar nuevas publicaciones. Se estima que solo el veinticinco por ciento de los manuscritos y dibujos heredados por Melzi se conservan en nuestros días en la forma de los famosos códices (Isaacson, 2017).

Según el relato de Vasari, la única fuente disponible sobre la muerte de Leonardo, este fallece en los brazos del rey Francisco I. En sus palabras: "el rey se acercó y le sostuvo la cabeza para ayudarlo y demostrarle su favor, así como para aliviar su malestar. Entonces el divino espíritu de Leonardo, reconociendo que no podía gozar de mayor honor, expiró en los brazos del rey" (Vasari, 2012). Este momento, probablemente idealizado, fue inmortalizado tres siglos después por Jean-Auguste-Dominique Ingres (figura 2). 
Este relato de la muerte de Leonardo ha sido cuestionado en su veracidad ya que existe el registro de que el rey Francisco I emitió una proclamación el 3 de mayo en la localidad de SaintGermain-en-Laye, a dos días de viaje de Amboise, lo que sugiere que no estuvo presente en la muerte de Leonardo el día anterior (Nicholl, 2005; Pedretti, 2011; Isaacson, 2017). Sin embargo, dicha proclamación está firmada por su canciller, y no por el rey, por lo que, considerando el aprecio que le tenía Francisco I y el evidente deterioro en la salud de Leonardo, aún cabe la posibilidad de que este momento sí haya sido real (Isaacson, 2017).

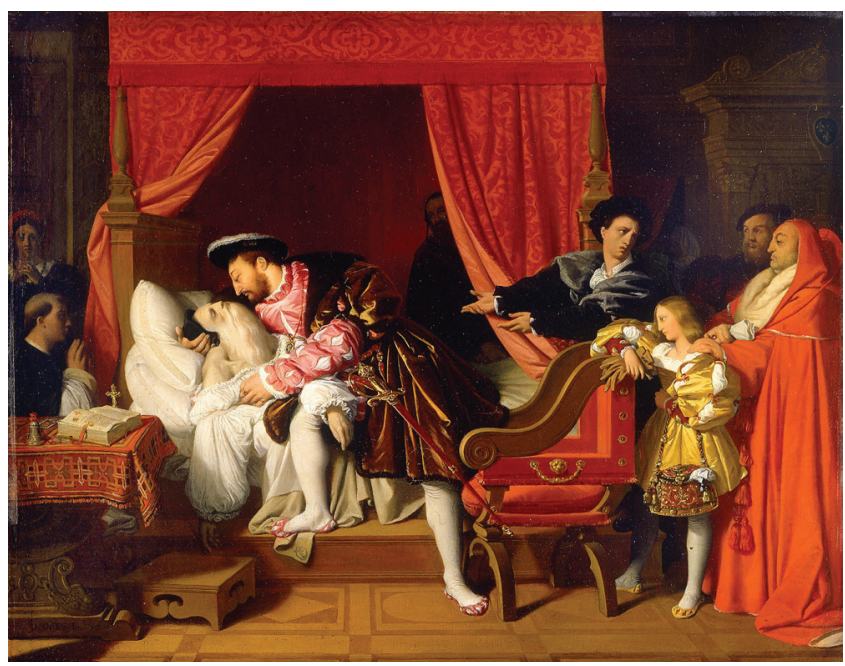

Figura 2: La muerte de Leonardo da Vinci, Jean Auguste Dominique Ingres (1818): Representación idealizada de la muerte de Leonardo da Vinci en los brazos del rey Francisco I, inspirada en el relato de Giorgio Vasari en "Las vidas de los más excelentes arquitectos, pintores y escultores italianos desde Cimabue a nuestros tiempos". [Museo de Bellas Artes "Petit Palais", Paris. Ministerio de Cultura de Francia].

Siguiendo sus designios, Leonardo fue enterrado en la iglesia de San Florentín de Amboise. Su tumba fue profanada cincuenta años después durante la revuelta de los hugonotes y luego, durante la revolución francesa, la iglesia fue destruida. (King, 2016). A principios del siglo XIX sus ruinas fueron demolidas por orden de Napoleón I y se puso un busto de mármol de Leonardo en su lugar (Nicholl, 2005; Pedretti, 2011; Isaacson, 2017). Las tumbas profanadas permitieron que las osamentas quedaran expuestas, de manera que era frecuente ver a niños jugando con ellas (King, 2016). Esto motivó a un jardinero de nombre Goujon a rescatar los huesos y enterrarlos en el lugar donde había estado el coro de la iglesia (King, 2016). Más de tres siglos después de la muerte de Leonardo, en 1863, excavaciones dirigidas por el escritor y político Arsène Houssaye, permitieron encontrar un esqueleto, una lápida incompleta y diversas monedas francesas e italianas, lo que le hicieron suponer, con muy poca rigurosidad técnica, que correspondían a Leonardo. Los restos fueron trasladados a la capilla San Huberto ubicada en el mismo palacio de Amboise y se puso una lápida con la inscripción de "supuestos restos" de Leonardo da Vinci (King, 2016).

Publicaciones han sugerido como prueba de que los restos pertenecen a Leonardo, el hecho de que en el esqueleto encontrado, el brazo derecho estaba en una posición viciosa, quizás secuela del ACV (Charlier \& Deo, 2017). Han existido iniciativas para exhumar los restos presuntos de la tumba de la capilla de San Huberto, pero el gobierno francés se ha resistido.

Con motivo de los 500 años de su muerte, un grupo de expertos creo el "Leonardo DNA project", siendo uno de sus objetivos el comparar los perfiles de ADN de los restos de la tumba presunta con los de descendientes de los diecisiete hermanos que tuvo Leonardo (Nelson, 2020). De exhumarse los restos de Leonardo y de confirmarse su autenticidad, sería una valiosa oportunidad para realizar una "autopsia molecular" para estudiar la presencia de polimorfismos genéticos que permitan valorar su riesgo de cáncer, de enfermedades cardiovasculares y otras enfermedades neurológicas. Adicionalmente, se podrían realizar determinaciones de metales pesados.

Independiente de a quien correspondan los restos de la capilla de San Huberto, y de la naturaleza de la enfermedad que afectó a Leonardo, queda claro que su muerte, al igual que su vida, estuvo rodeada de un halo de misterio. De lo que hay certeza es que esos últimos años de su vida, fueron los más tranquilos, donde se sintió más valorado y respetado, y donde no tuvo la presión de hacer obras por encargo que le persiguió durante toda su vida.

Su enfermedad, presumiblemente neurológica y progresiva, lo encontró rodeado de gente que le amaba y le admiraba, como lo fueron Francesco Melzi y el rey Francisco I. Los tres compartieron largos meses, donde de seguro Leonardo, forzosamente calmado e intuyendo una muerte próxima, influyó profundamente en el rey y fue instruyendo a Melzi, no solo como el buen discípulo que fue, sino como su albacea y administrador de su legado.

\section{Conclusión}

Leonardo hoy sigue siendo un personaje muy actual e inabarcable. Su obra es estudiada exhaustivamente, y son frecuentes las nuevas lecturas, revisiones y redescubrimientos. En ese contexto, todo en torno a su figura se encuentra rodeado por un cierto halo de misterio, despertando interés y curiosidad tanto en el público general como en el mundo académico.

En particular, con respecto a sus últimos años, existen registros históricos confiables que dan cuenta de que efectivamente la movilidad de su 
brazo derecho se encontraba disminuida, limitando su capacidad para pintar, y de que luego su movilidad general y su condición global se fue deteriorando, quizás de una manera "escalonada" propia de los ACVs recurrentes. La hipótesis del veganismo como factor de riesgo del presunto ACV de Leonardo es solo una especulación con poco sustento, ya que desconocemos su perfil de riesgo global, en términos de los factores modificables con mayor riesgo atribuible para ACV como hipertensión arterial, diabetes mellitus o dislipidemia. Otros diagnósticos alternativos como una lesión de nervio periférico son planteables, no obstante, no explican el deterioro posterior descrito por Vasari. Una enfermedad de la neurona motora o una intoxicación por metales pesados son posibilidades interesantes y hasta la fecha no consideradas por biógrafos e investigadores.

Leonardo, incapaz de acometer nuevos proyectos, se dedicó a estimular el desarrollo del Renacimiento en Francia mediante su influencia sobre el rey Francisco l y a la vez, encontró en Francesco Melzi un albacea digno de su obra. En gran medida gracias a ambos, y a la estrecha relación que tuvieron con Leonardo en esos años de calma, prosperidad y de una muerte que se le anunciaba, es que el legado de Leonardo ha llegado hoy hasta nosotros.

\section{Contribuciones y reconocimientos:}

No existen fuentes de financiamiento. El autor declara no tener ningún conflicto de interés

\section{Referencias}

Bambach C. (2003). Leonardo, Left-Handed Draftsman and Writer. In Leonardo da Vinci Master Draftsman, exhibition catalogue, ed. Bambach C, pp 31-58. The Metropolitan Museum of Art, New York; Yale University Press, New Heaven and London.

Bambach C. (2019). Leonardo da Vinci Rediscovered. Yale University Press, London.

Campanozzi LL, Benvenuto D, Guarino MPL. Lauri G \& Tambone V. (2020). Leonardo da Vinci's advice on public health. Lancet 395, e16.

Campbell T. (2017). A plant-based diet and stroke. Journal of Geriatric Cardiology 14, 321-326.

Coralli A \& Perciaccante A. (2016). Leonardo's recurrent stroke? Lancet Neurology 15, 667.

Cummins N. (2017). Lifespans of the European Elite, 800-1800. The Journal of Economic History 77, 406-439.

Charlier P. \& Deo S. (2017). A physical sign of stroke sequel on the skeleton of Leonardo da Vinci? Neurology 88, 1381-1382. de Campos D, Coutinho Rodrigues D. \& Buso L. (2019). The right hand of Leonardo da Vinci (1452-1519): ulnar or median nerve palsy? Journal of Royal Society of Medicine 112, 452.

Forcellino A (2012). Raphael: a passionate life. Wiley, New Jersey.

Greenspan P, Heinz G \& Hargrove JL. (2008). Lives of the artists: differences in longevity between old master sculptors and painters. Age and Ageing 37, 102-104.

Isaacson W. (2017). Leonardo da Vinci: the biography. Simon \& Schuster, New York.

Kemp M. \& Pallanti G. (2017). Mona Lisa: The People and the Painting. Oxford University Press, Oxford.

Kernan WN, Ovbiagele B, Black HR, Bravata DM, Chimowitz MI, Ezekowitz MD, Fang MC, Fisher M, Furie KL, Heck DV, Johnston SC, Kasner SE, Kittner SJ, Mitchell PH, Rich MW, Richardson D, Schwamm LH, Wilson JA. el al. (2014). Guidelines for the prevention of stroke in patients with stroke and transient ischemic attack: a guideline for healthcare professionals from the American Heart Association/ American Stroke Association. Stroke 45, 2160-2236.

King R. (2016). Leonardo's Bones: Myth, History, and Evidence. Human Evolution 31, 133-147.

Lazzeri D. \& Rossi C. (2019). The right hand palsy of Leonardo da Vinci (1452-1519): new insights on the occasion of the 500th anniversary of his death. Journal of Royal Society of Medicine 112, 330-333.

Ma C, Gurol ME, Huang Z, Lichtenstein AH, Wang X, Wang Y, Neumann S, Wu S. \& Gao X. (2019). Low-density lipoprotein cholesterol and risk of intracerebral hemorrhage: A prospective study. Neurology 93, e445-e457.

McCarty MF. (2003). IGF-I activity may be a key determinant of stroke risk--a cautionary lesson for vegans. Medical Hypotheses 61, 323-334.

McManus IC. (1975). Life expectation of Italian Renaissance artists. Lancet 1, 266-267.

McManus IC. \& Drury H. (2004). The handedness of Leonardo da Vinci: a tale of the complexities of lateralisation. Brain and Cognition 55, 262-268.

Meschia JF, Bushnell C, Boden-Albala B, Braun LT, Bravata DM, Chaturvedi S, Creager MA, Eckel RH, Elkind MS, Fornage M, Goldstein LB, Greenberg SM, Horvath SE, ladecola C, Jauch EC, Moore WS, Wilson JA. et al. (2014). Guidelines for the primary prevention of stroke: a statement for healthcare professionals from the American Heart Association/American Stroke Association. Stroke 45, 3754-3832. 
Muntz E.(1898). Leonardo da Vinci, Artist, Thinker and Man of Science. W. Heinemann, London.

Naghshi S, Sadeghi O, Willett WC \& Esmaillzadeh A. (2020). Dietary intake of total, animal, and plant proteins and risk of all cause, cardiovascular, and cancer mortality: systematic review and dose-response meta-analysis of prospective cohort studies. BMJ 370, m2412.

Nelson K. (2020). Leonardo da Vinci DNA project. J. Craig Venter Institute. Accedido en https://www.jcvi.org/research/leonardo-da-vinci-dna-project el 06 de Octubre de 2020.

Nicholl C. (2005). Leonardo. El vuelo de la mente. Taurus, Madrid.

Öztürk Ş, Altieri M. \& Troisi P. (2010). Leonardo Da Vinci and stroke - vegetarian diet as a possible cause. Frontiers of Neurology and Neuroscience 27, 1-10.

Özturk Ş. (2009). Leonardo Da Vinci (1452-1519) as a stroke victim: hemiparesis a result of a vegetarian diet? Journal of Medical Biography 17, 7.

Pedretti C. (2011). Leonardo da Vinci in France. CB Publishers, Poggio a Caiano.

Remuzzi G. \& Ricciardi W. (2019). Why and how did Leonardo happen: the Renaissance context. Lancet 393, 1396-1397.

Peller S. (1947). Studies on mortality since the Renaissance. Bulletin of the History of Medicine 21, 51-101.

Reinke KR.(1993). Leonardo Da Vinci's right-to-left "mirrored" writing revisited. Annals of Plastic Surgery 30, 89-92.
Riancho J, Bosque-Varela P, Perez-Pereda S, Povedano M, de Munaín AL. \& Santurtun A. (2018). The increasing importance of environmental conditions in amyotrophic lateral sclerosis. International Journal of Biometeorology 62, 1361-1374.

Richter JP. (1977). The Literary Works of Leonardo da Vinci, Compiled and Edited from the Original Manuscripts by Jean Paul Richter: Commentary by Carlo Pedretti. Vol 2. University of California Press, Berkeley and Los Angeles.

Schott GD. (1979). Some neurological observations on Leonardo da Vinci's handwriting. Journal of Neurological Sciences 42, 321-329.

Shi Z, Liu S, Guan Y, Zhang M, Lu H, Yue W, Zhang B, Li M, Xue J. \& Ji Y. (2018). Changes in total homocysteine levels after acute stroke and recurrence of stroke. Scientific Reports 8, 6993.

Tambone V, Lauri G, Guarino MPL, Campanozzi LL. \& Ciccozzi M. (2019). Leonardo's folio 730 recto: lessons for the medical humanities. Lancet 393, 1411-1412.

Tong TYN, Appleby PN, Bradbury KE, Perez-Cornago A, Travis RC, Clarke R. \& Key TJ. (2019). Risks of ischaemic heart disease and stroke in meat eaters, fish eaters, and vegetarians over 18 years of follow-up: results from the prospective EPIC-Oxford study. BMJ 366, 14897.

Vasari G. (2012). Las vidas de los más excelentes arquitectos, pintores y escultores italianos desde Cimabue a nuestros tiempos. Cátedra, Madrid.

Wallace W.(2011). Michelangelo: The Artist, the Man and his Times. Cambridge University Press, Cambridge. 